\title{
Accurate Determination of the Deadtime and Recovery Characteristics of Geiger-Müller Counters
}

\author{
By Lovis Costrell
}

\begin{abstract}
A brief description of the theory of operation of Geiger-Müller counters is presented, with special emphasis on the deadtime and recovery characteristics. An electronic gating instrument is described that has been developed for the determination of deadtime and recovery characteristics to an accuracy of 1 percent or 2 microseconds. Experimental data are presented showing variation of deadtime and recovery time with counter pressure and overvoltage.
\end{abstract}

\section{Introduction}

Geiger-Müller counters are extensively used in the measurement of radiation from atomic nuclei. A knowledge of their characteristics is, therefore, essential in interpreting the data obtained. The deadtime and the recovery characteristics of Geiger counters are of interest not only because they are important factors in determining the errors that occur, but also because they arc intimately associated with the discharge mechanism. An electronic instrument has been designed and constructed for the accurate determination of the deadtime and recovery characteristics of Geiger counters. The pulses initiate "gates" that can be set to predetermined durations so as to permit registering of pulses that follow the initiating pulses within the time that the gate is open. A few instruments, ${ }^{1234}$ quite different in design, though somewhat similar in function, have been developed for determination of spurious

\footnotetext{
1 R. L. Driscoll, M. W. Hodge, and A. E. Ruark, Rev. Sci. Inst. 11, 241 (1940). The instrument described is suitable only for intervals greater than $0.1 \mathrm{sec}$.

${ }^{2}$ Arthur Roberts, Rev. Sci. Inst. 12, 71 (1941). The instrument described has a range of $10^{-4}$ to $0.3 \mathrm{sec}$. It utilizes gas tubes preceding a pulse lengthener and a Rossi coincidence circuit. Counter characteristics and resolving time have been investigated with this instrument. Counters giving even a small percentage of multiple pulses ar e apparently readily detected.

${ }^{3}$ F. L. Davis and L. F. Curtiss, J. Research NBS 29, 405 (1942) RP1509. The instrument described has a range of $3 \times 10^{-5}$ to 0.2 sec. and is used primarily for testing for multiple pulses in alcohol-argon counters. The data obtained permit determination of the resolving time.

${ }^{4}$ S. C. Curran and E. R. Rae, Rev. Sci. Instr. 18, 871 (1947). The instrument described is a modification of the Roberts instrument. A variakle time delay is included that permits accurate determination of th 3 number of spurious counts that occur.
}

pulses and resolving time. The instrument described herein uses no relays or gas tubes, hard vacuum tubes being used exclusively. The instrument has been designed specifically for the determination of the deadtime and recovery characteristics of Geiger counters and has high accuracy when used for this purpose. An accuracy of 1 percent or 2 microseconds (whichever gives the larger error) is obtained.

\section{Deadtime, Recovery Time, and Resolving Time}

When an ionizing event occurs in a GeigerMüller counter, the negative electrons are attracted to the positive wire. Since the electric field in the vicinity of the wire is extremely high, the electrons released in the initial ionization acquire sufficient velocity to release additional electrons from neutral atoms that lie in their paths. The electrons so released dislodge additional electrons as they travel toward the wire. This cumulative ionization is known as a Townsend avalanche. The electrons that reach the wire contribute a negative electric charge to the wire. This charge is responsible for the negative voltage pulses obtained from the counter.

The electrons, being light and therefore highly mobile, reach the wire within a fraction of a microsecond after the ionization has occurred. The positive ions proceed at the same time toward the negative cylinder. However, being 


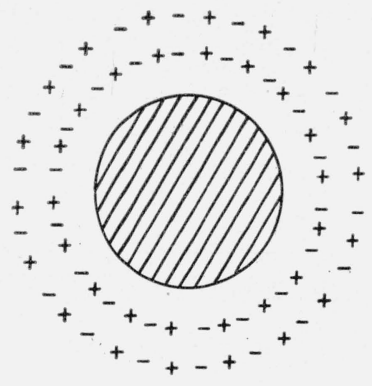

(a)

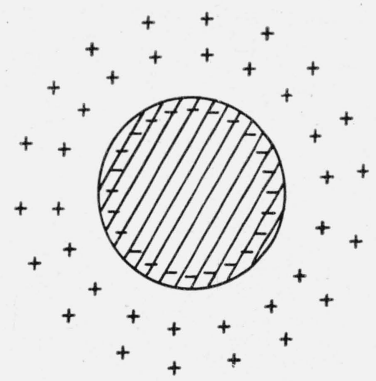

(b)
FIGURE 1. Sheath of electrons and positive ions about positive wire of Geiger counter.

a, Immediately after avalanche; b, a fraction of a microsecond later.

heavy particles, they proceed at a slow rate compared to the fast-moving electrons. In addition, they have a further handicap in that they have a considerably longer distance to travel than do the electrons. This is the case since practically all of the secondary electrons are produced in the high field region very close to the wire. Figure 1 , a, shows a simplified version of the region very close to the wire immediately after the avalanche has occurred. A fraction of a microsecond later the electrons have reached the wire, whereas the positive ions are still stranded about the wire, as shown in figure $1, b$. This sheath of positive ions forms a shield about the wire, thus reducing the field intensity to less than the value necessary for Geiger action. A particle entering the counter at this time is thus unable to produce an avalanche. This sheath of positive ions moves slowly outward, finally traveling far enough from the wire so that the electric field about the wire increases to a value sufficient to permit Geiger action to resume. ${ }^{5}$ However, since the positive ion sheath is still unneutralized within the counter, its shielding effect does not permit the normal electric field intensity to exist about the wire. Therefore, pulses of reduced size will result from particles entering the counter very shortly after the positive ions have moved an amount sufficient to permit the resumption of Geiger action. As the ions move toward the cylinder, the electric field intensity near the wire increases, finally reaching its full value when the ions have reached the cylinder. Thus, full size pulses cannot occur until the positive ions have reached the cylinder. The action of polyatomic gas molecules in quench-

5 The theory of the action of the positive ion sheath in this manner is due to C. G. and D. D. Montgomery, J. Franklin Inst. 231, 509 (1941). ing the discharge also plays an important part in the counter operation, though this action will not be discussed here.

Physically, the deadtime is the time required for the positive ions to travel far enough from the wire to permit the resumption of Geiger action, and the recovery time is the time required for the positive ions to reach the counter wall. Functionally, the deadtime and recovery time can be defined as follows:

Deadtime: The time interval after a pulse has occurred during which the counter is insensitive to further ionizing events.

Recovery time: The time interval that must elapse after a pulse has occurred before a full-size pulse can again occur.

Since a pulse of finite size is required to operate a detecting circuit, the resolving time of the counter and its accessories (amplifying and scaling circuits, etc:) exceeds the deadtime of the counter.

The resolving time can be defined as follows:

Resolving time: ${ }^{6}$ The minimum time interval by which two pulses must be separated to be detected as separate pulses by the counter and its accessories.

Stever ${ }^{7}$ has described a method for determining the deadtime and recovery characteristics of Geiger counters by means of a cathode ray oscilloscope. The accuracy is adequate for most purposes. For best results a time exposure photograph should be taken of the oscilloscope screen pattern, though the deadtime and the recovery curve can be observed visually, especially if a high counting rate and persistent screen are used. This method will be described as it gives an excellent picture of the deadtime and recovery phenomena. The pulses from the counter are applied to the $Y$-axis amplifier of the oscilloscope and also made to trigger the sweeps. (Some oscilloscopes, such as the DuMont type 248 , have driven sweep circuits that can be triggered externally and are well suited for this purpose. The 1,000microsecond sweep on the DuMont type 248 oscilloscope gives good results.) Consider counter pulses applied to an oscilloscope in this manner

\footnotetext{
${ }^{6}$ Resolving time has been a loosely used term, frequently being applied to a counter. Actually, the resolving time is a function of the input sensitivity of the scaler, as well as of the deadtime and recovery characteristic of the counter.

${ }^{7}$ H. G. Stever, Phys. Rev. 61, 38 (1942).
} 
with a sweep of 1,000-microseconds duration. If the counting rate is 100 counts per second, the average spacing between pulses will be 10,000 microseconds. Thus, after a sweep has been initiated by a pulse, there is a probability of approximately one out of 10 that another pulse will appear on the screen. Such a pulse is shown as pulse $\mathrm{P} 1$ in figure 2, a. During some other sweep a pulse, $\mathrm{P} 2$, as shown in figure $2, \mathrm{~b}$, will appear. On still another sweep, a pulse P3 of figure 2, c, will appear on the screen. If a time exposure photograph is taken of the screen, random pulses appearing at various instances will be recorded on the film, giving the pattern shown in figure 2 , d. The deadtime, recovery time, and resolving time are indicated in figure $2, \mathrm{~d}$.

\section{Resolving Time Errors}

Since nuclear disintegrations occur in a random manner, there is a finite probability of two disintegrations occurring within any given time interval. Since the counter is dead for a short period after a pulse has occurred, some of the disintegrations will not be detected. This will introduce a discrepancy between the number of particles entering the counter and the number of counts recorded. The resulting error can be calculated as follows:

The counter is insensitive for a time, $t_{r}$, after each pulse, where $t_{r}$ is the resolving time of the counter in seconds. On the average, the counter is sensitive for a period $\left(1 / a-t_{r}\right)$ after each pulse, where $a$ represents the true number of particles detected per second. For values of $t_{r}<<1 / a$, the sensitive time is, therefore, approximately $1 / a$. Therefore,

$$
\frac{\text { insensitive time }}{\text { sensitive time }}=\frac{t_{r}}{1 / a}=a t_{r} .
$$

For values of $a t_{\tau}<<1 / a, n$ (the observed number of particles detected per second) is approximately equal to $a$. Therefore,

$$
\text { Percentage error }=100 \mathrm{nt}_{r} \text {. }
$$

It is readily seen that a counting rate of 100 counts per second, using a counter with a resolving time of 300 microseconds, will result in a resolving time error of 3 percent, whereas the same counting rate with a counter with a 1,000 -microsecond re-

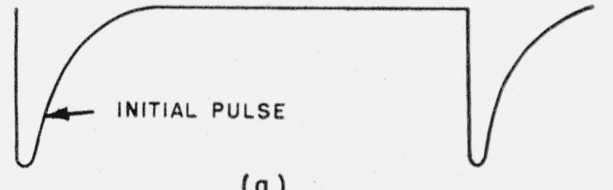

(a)
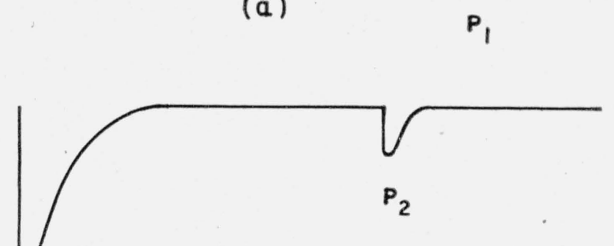

(b)

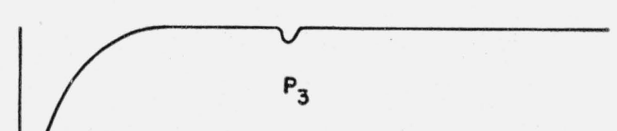

(c)

INPUT SENSITIVITY OF DETECTING CIRCUIT]

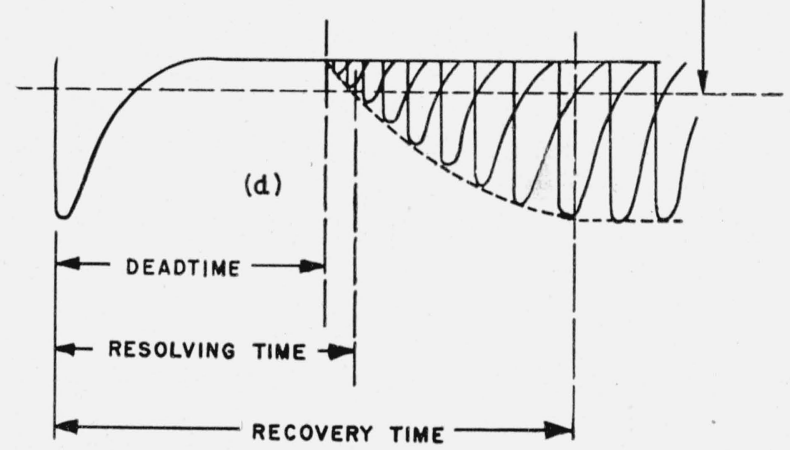

Figure 2. Deadtime, recovery time, and resolving time. $\mathrm{P} 1, \mathrm{P} 2$, and $\mathrm{P} 3$ are random pulses.

solving time will result in a resolving time error of approximately 10 percent. If the counter is calibrated against a source of the same intensity as the test sample, the resolving time error will be automatically compensated for. However, this is not the case when the calibrating standard and the test sample vary appreciably in intensity. Corrections can, however, be made for the resolving time error by the use of eq 1 .

\section{Statistical Analysis}

Determination of the deadtime and recovery characteristics of Geiger counters by consideration of the number of pulses falling within given time intervals necessitates analysis of the statistical behavior of random events. Let 
$N=$ Accepted counts per second (particles detected per second that occur within given time intervals after preceding detected particles).

$a=$ True particles detected per second.

$n=$ Observed particles detected per second (true particles detected per second minus coincidence loss).

$t=$ time in seconds.

$t_{d}=$ deadtime in seconds.

$t_{g}=$ gating time in seconds.

$t_{80}, t_{50}, t_{20}$, etc. $=$ time in seconds to recover to 80 , 50, 20 percent, etc.

The instrument described later accepts only pulses occurring between the end of the deadtime and the end of the gating period. The instrument is thus sensitive only during the time interval $\left(t_{g}-t_{d}\right)$. The probability that no countable particle will appear during this time interval is $e^{-a\left(t_{g}-t d\right)}$. For values of $a\left(t_{g}-t_{d}\right)$ much less than unity, the average number of counts per second that will occur in the time interval $\left(t_{g}-t_{d}\right)$ is given by eq 2 .

$$
N=a\left[1-e^{-a\left(t_{g}-t d\right)}\right] .
$$

Figure 3 is a plot of eq 2. The time-axis intercept of the curve gives the deadtime.

Equation 2 can be simplified as follows:

Expanding eq 2

$$
N=a\left[1-1+a\left(t_{g}-t_{d}\right)-\frac{a^{2}\left(t_{g}-t_{d}\right)^{2}}{2 !}+\ldots .\right]
$$

Ignoring terms higher than first order,

$$
N=a^{2}\left(t_{g}-t_{d}\right) .
$$

Since $n$ is approximately equal to $a$,

Rearranging,

$$
N=n^{2}\left(t_{g}-t_{d}\right) .
$$

$$
t_{d}=t_{g}-\frac{N}{n^{2}}
$$

Best results are obtained by determining a few points on the straight-line portion of the curve of figure 3 and extrapolating the curve to give the deadtime. If only pulses above a certain size are accepted by the instrument, a curve similar to figure 3 , but displaced somewhat to the right, will

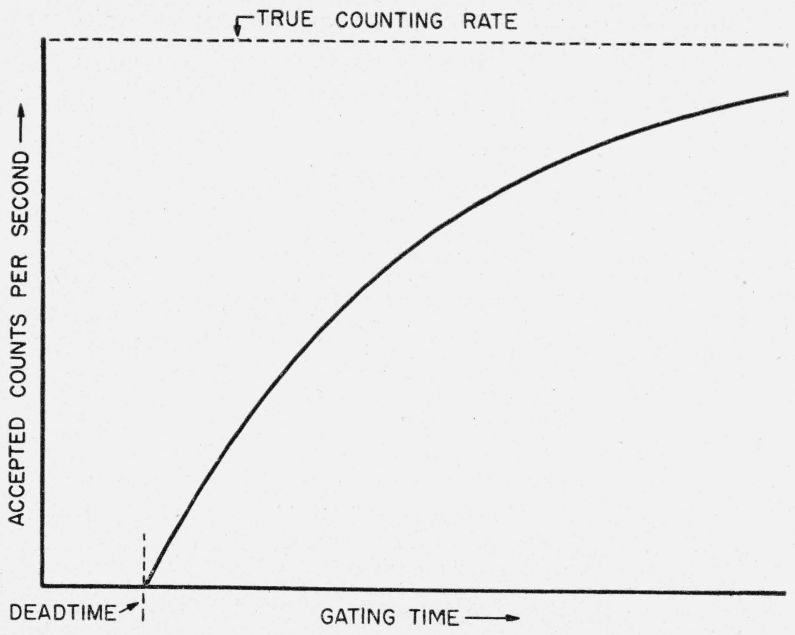

FIGURE 3. Accepted counts per second versus gating time.

be obtained. The time axis intercepts of each of these curves represents the time for recovery to the minimum pulse size accepted. Figure 4 shows such curves for various minimum pulse sizes. The data from figure 4 can be replotted to give the complete recovery curve of the counter, as shown in figure 5 .

\section{Description of Instrument}

The instrument developed for the determination of the deadtime and recovery characteristics of Geiger counters is shown in block form in figure 6 . Figure 7 is a schematic diagram of the circuit.

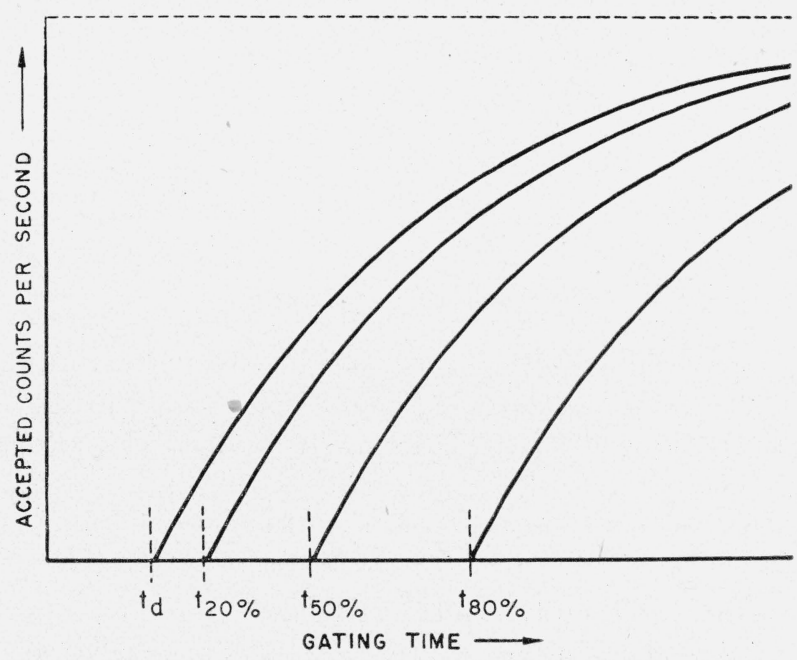

FIGURE 4. Accepted counts per second versus gating timé for various degrees of recovery.

$t_{d}$, Deadtime; $t_{20 \%}$, time to recover to 20 percent; $t_{50 \%}$, time to recover to 50 percent; $t_{80 \%}$, time to recover to 80 percent. 


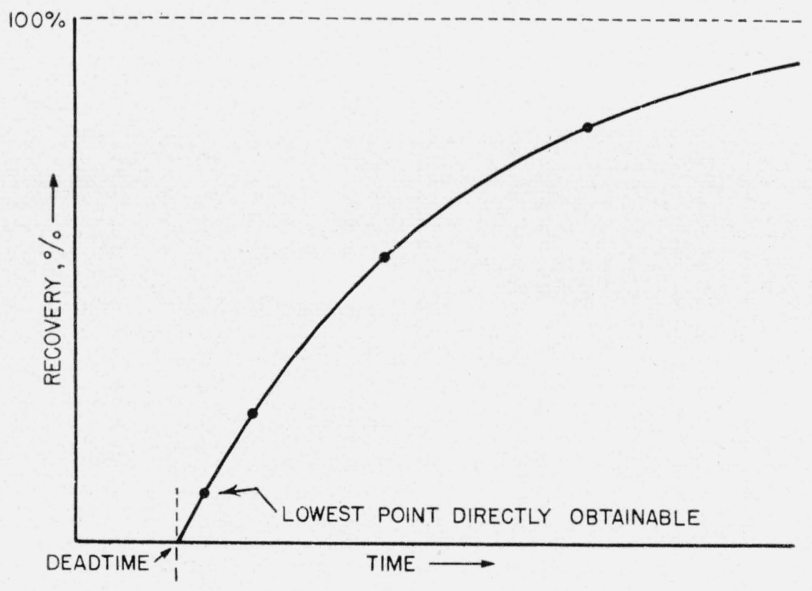

Figure 5. Recovery curve of Geiger counter.

The random pulses from the Geiger counter are fed into a pulse amplifier, then into a pulse height selector, then through pulse shaping circuits to give sharp negative pulses of uniform height. These sharp negative pulses then follow two paths; one by the way of an amplifier stage to a heavily biased amplifier, the output of which connects to the input of a conventional scaler, and the other through a 15-microsecond delay circuit to a "one-shot" gating multivibrator. Figure 8 is a schematic diagram of the delay circuit and gating multivibrator. The gating multivibrator controls the bias on the biased amplifier so as to permit pulses that follow other pulses by less than the gating time to go through the biased amplifier and actuate the scaling and recording circuits.
The gate is delayed 15 microseconds with respect to the initiating pulse, so that the pulse initiating the gate will be completed before the gate opens. Thus a pulse cannot pass through the gate that it initiates, though succeeding pulses that follow within the gating time are accepted. In the pulse height selector stage the bias is varied so as to discriminate against pulses of less than a given height. This permits determination of the recovery curve of the counter. A simple visual pulse-size indicator, as shown in figure 9, is incorporated in the instrument to permit ready adjustment of the input potentiometer.

The counter pulses are applied through potentiometers $R_{2}$ and $R_{3}$ (fig. 7 ) to the control grid of tube $\mathrm{T} 1$ which is a conventional class $\mathrm{A}$ resistance coupled amplifier. The output of this stage is then applied to the pulse size indicator and to the control grid of the pulse height selector. The pulse height selector, tube T2, is a sharp cutoff amplifier in which the control grid bias is adjusted by means of potentiometer $R_{9}$ to obtain the desired pulse size discrimination. (A biased diode would also be satisfactory for this application.) This permits determination of the recovery curve, as shown in figure 5. The bias voltage is indicated on the voltmeter shown in the grid circuit of tube T2. The output pulses of the pulse height selector vary considerably in size. The pulses are, therefore, amplified by tube T3a and T3b, and then clipped by tube T4a so that the pulses at the clipper output are of uni-
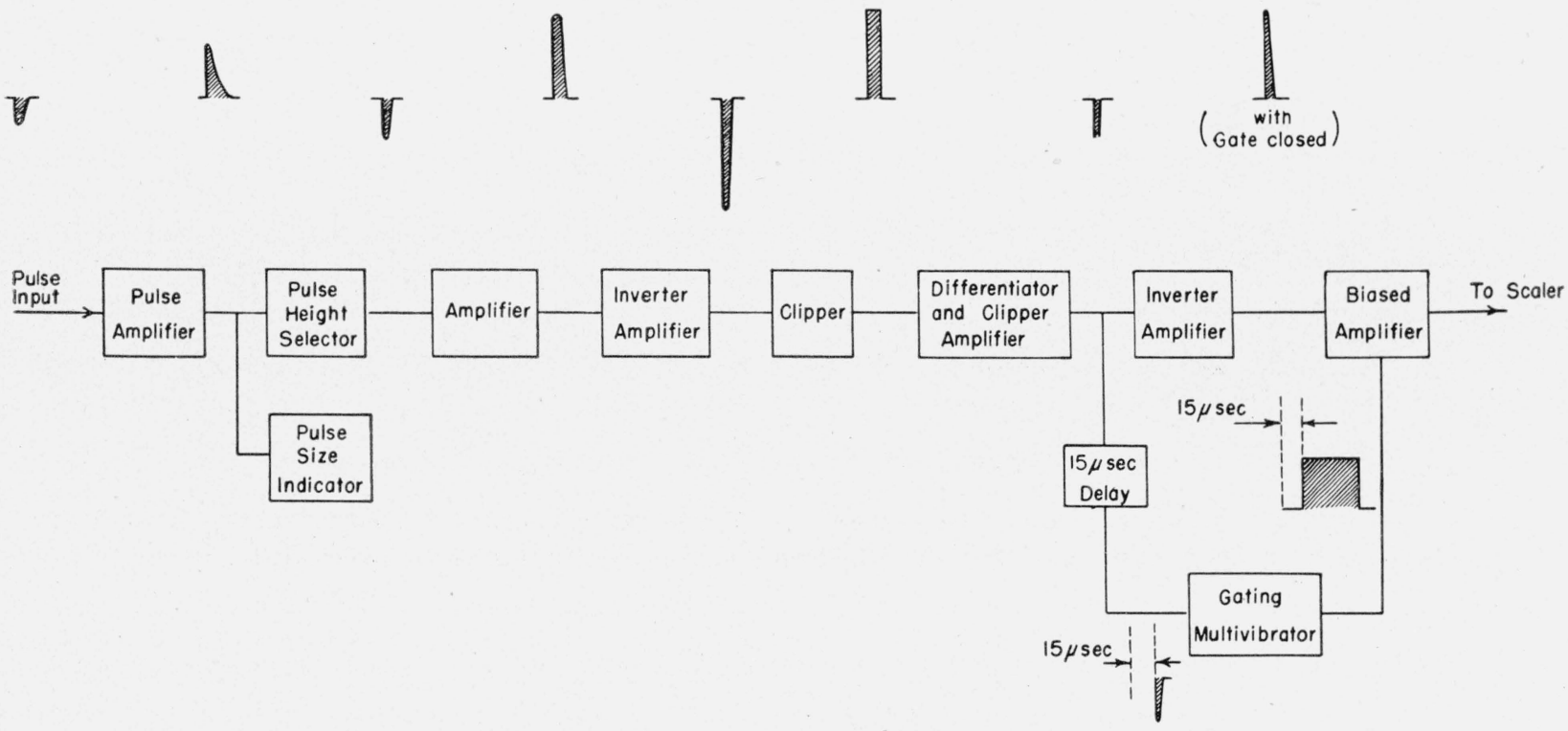

FiguRE 6.-Block diagram of instrument. 


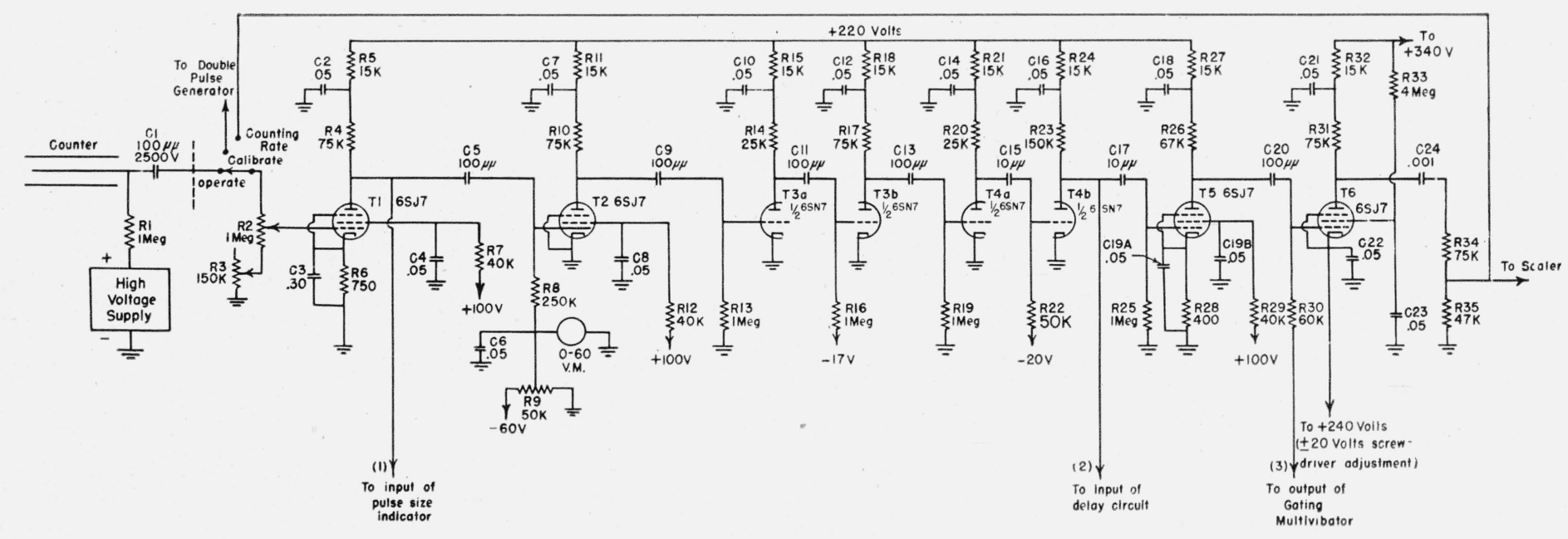

FigURE 7. Schematic diagram of instrument.

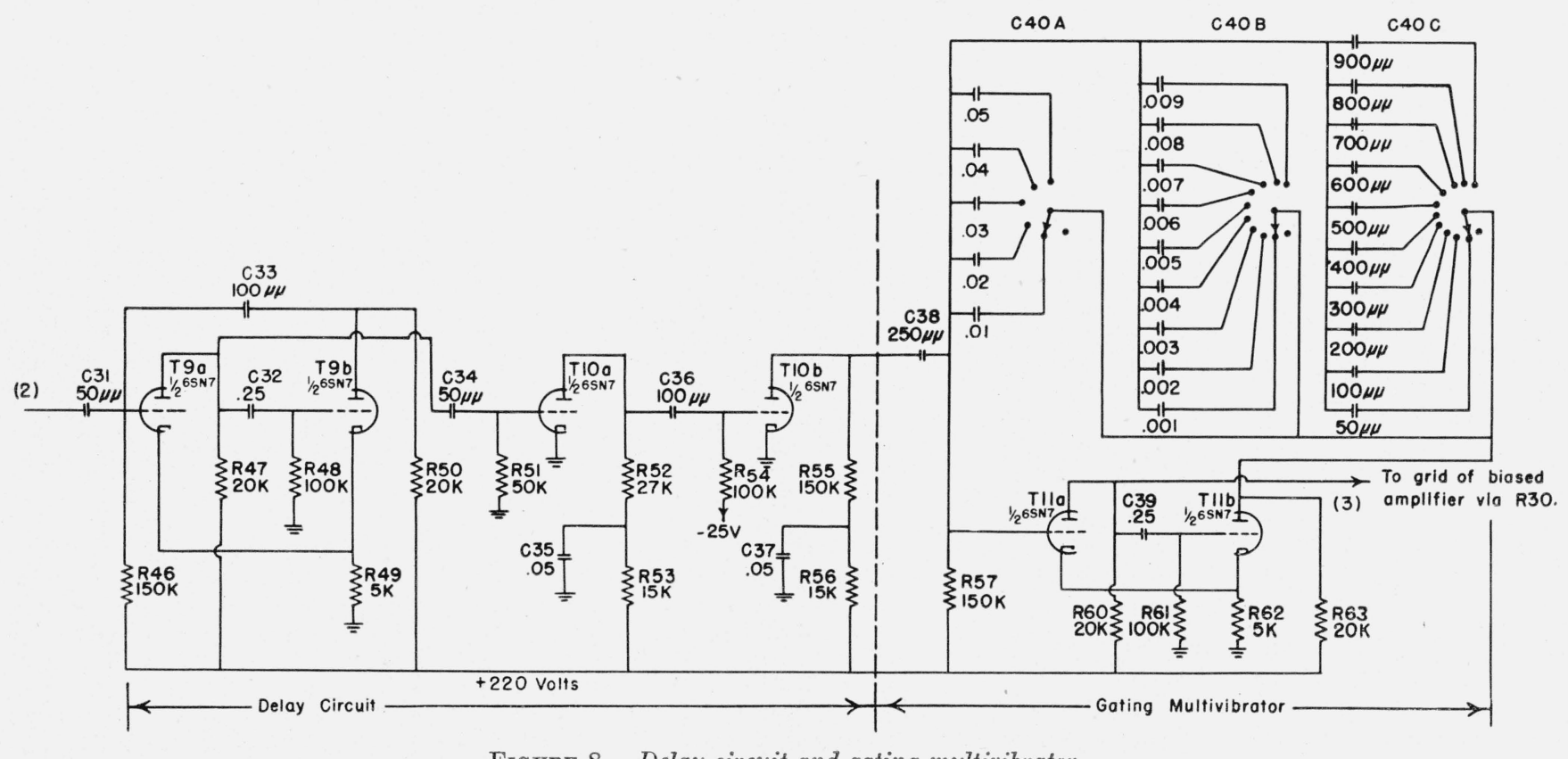

FIGURE 8. Delay circuit and gating multivibrator. 


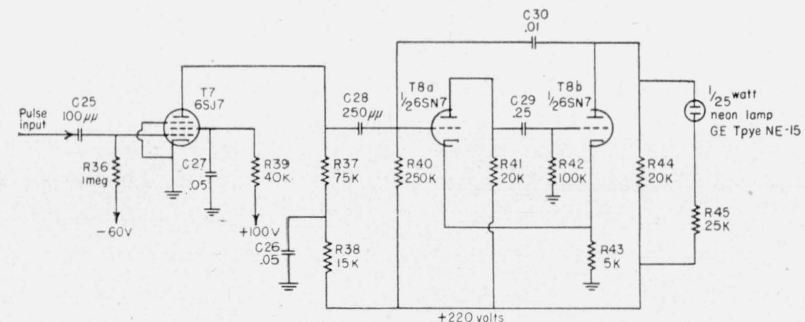

Figure 9. Pulse size indicator.

form height. The positive output pulses of the clipper are differentiated by the $\mathrm{RC}$ network in the grid circuit of tube T4b. The trailing edge pulse is clipped by means of the grid bias, whereas the leading edge is retained. The sharp negative pulses at the output of $T 4 b$ trigger the delay circuit, that in turn triggers the gating multivibrator. The pulses from $T 4 b$ are also inverted and amplified by $\mathrm{T} 5$ to produce large positive output pulses for application to the biased amplifier T6.

The biased amplifier, T6, has a high negative bias so as to block the positive pulses from T5. Since the control grid of T6 is connected to the plate of the normally conducting half of the gating multivibrator, it is at a positive potential of approximately $80 \mathrm{v}$ with respect to ground. Therefore, the negative bias of approximately $120 \mathrm{v}$ is obtained by maintaining the cathode at approximately $200 \mathrm{v}$ positive. When the gating multivibrator "opens the gate", the bias is reduced sufficiently to permit the acceptance of the positive pulses from the output of T5, as shown in figure 10. The pulses at the output of the biased amplifier T6 are then applied to the scaling circuit, where they register as accepted counts.

The negative pulses at the output of T4b trigger the one-shot multivibrator, tube T9a and T9b of figure 8, producing rectangular pulses of approximately 15-microseconds duration. These rectangular pulses are differentiated, amplified, and then clipped. The pulse resulting from the leading edge of the 15-microsecond pulse is eliminated in the clipping operation, while the trailing edge pulse is retained. The pulse retained is thus delayed approximately 15 microseconds with respect to the initiating pulse and is used to trigger the gating multivibrator. ${ }^{8}$

\footnotetext{
${ }^{8}$ It is planned to replace the 15 -microsecond delay circuit by a variable time delay circuit in order to increase the versatility of the instrument.
}

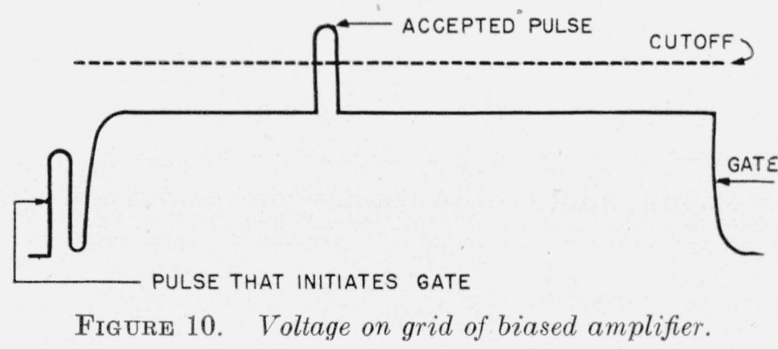

The gating multivibrator, tube T11a and T11b of figure 8 , is a one-shot multivibrator with a plateau adjustable between approximately 30 - and approximately 5,000-microsecond duration. T11a is normally conducting. It is rendered nonconducting by a negative pulse and remains nonconducting for a time determined by the values of capacitors $\mathrm{C} 40$ and resistor R57. When T11a becomes nonconducting, its plate voltage rises and assumes the value of the supply voltage. Thus, a rectangular positive pulse appears at the output of the gating multivibrator, when triggered. This rectangular pulse is applied to the grid of the biased amplifier T6, opening the gate by decreasing the bias to such a value that pulses from T5 that occur while the gate is open, are accepted by the biased amplifier and, therefore, actuate the scaling circuit. It will be noted from figure 10 that the gate begins approximately 15 microseconds after the initiating pulse, so that a pulse cannot pass through the gate that it initiates. Since the 15 microseconds is considerably shorter than the deadtime of Geiger counters of the types now in general use, none of the pulses that should register are blocked because of the delay.

The pulse size, indicator, figure 9, consists simply of a biased amplifier and a one-shot multivibrator with a neon lamp across the load resistor of the normally nonconducting triode. When the input pulses are of sufficient amplitude to overcome the bias on the biased amplifier $\mathrm{T} 7$ and trigger the multivibrator T8a and T8b, the voltage across the load resistor $\mathrm{R} 44$ rises to approximately $100 \mathrm{~V}$ for approximately 2,500 microseconds. Since the neon lamp starts conducting at approximately $70 \mathrm{v}$, this causes the lamp to conduct. The lamp is essentially a constant-voltage device, maintaining a voltage of approximately $65 \mathrm{v}$ while conducting. Thus, the voltage across the $25 \mathrm{~K}$ resistor is approximately $35 \mathrm{v}$ and the current through the lamp and the resistor approximately 1.4 mils. This current is drawn for approxi- 
mately 2,500 microseconds for each pulse, producing bright flashes. With counting rates of the order generally used (approximately 100 pulses per second) the flashes appear so frequently that the lamp appears to be on continuously when flashing. The bias of $\mathrm{T} 7$ is permanently adjusted so that the lamp will begin to flicker when the input pulses are of the proper size. A biased diode would also be satisfactory for this application, in lieu of the pentode used for T7.

Any accurate double pulse generator can be used to calibrate the instrument. Conventional double pulse generators utilize blocking oscillators or one-shot multivibrators. Actually, a double pulse generator utilizing a shock-excited oscillator was used. The shock-excited oscillator is sharply attenuated by means of resistance across the tuned circuit. The oscillations are then clipped to eliminate all but two half cycles, and these two are shaped to give the double pulses desired. The spacing between the two pulses comprising a pair is determined primarily by the constants in the cathode circuit of the shock-excited oscillator. The repetition frequency is the repetition frequency of the square wave generator that controls the shock-excited oscillator.

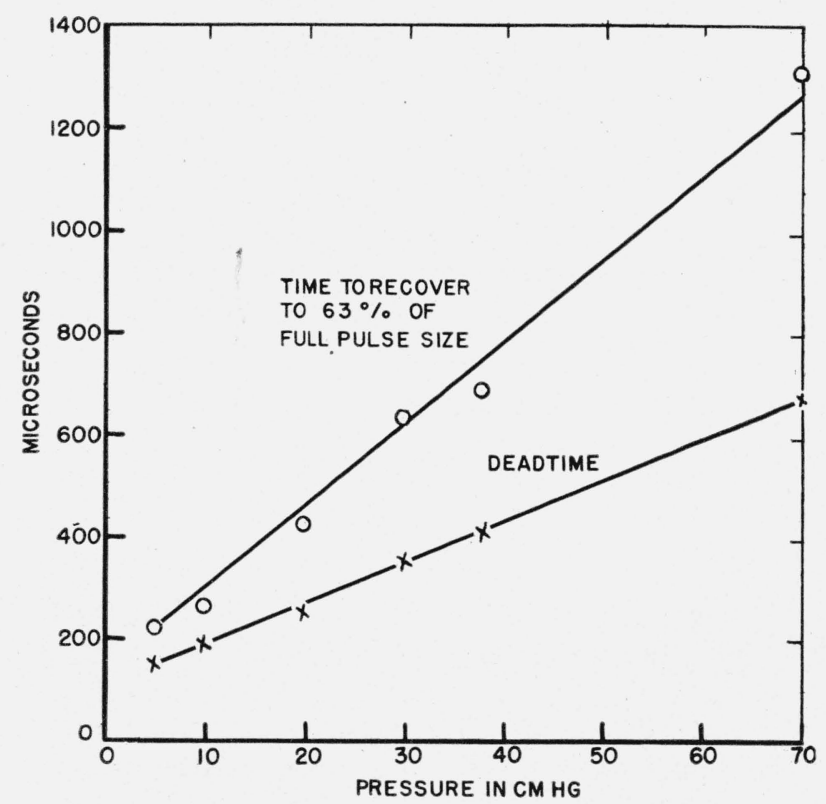

FIGURE 11. Variation of deadtime and recovery time of mica window argon-alcohol counters with variation in total pressure.

Counters filled with alcohol at pressure of $12 \mathrm{~mm}(\mathrm{hg})$ and remainder argon. Overvoltage $=75 \pm 5 \mathrm{v}$.

\section{Typical Data}

The instrument is currently being used in the routine determination of the deadtime and recovery characteristics of Geiger counters.

Investigations have been made of the influence of pressure and overvoltage on deadtime and recovery time. The counters tested were of the mica window type and contained argon, to which had been added alcohol at a pressure of $12 \mathrm{~mm}$ of mercury. The results of these investigations are shown in figures 11 and 12 . It will be noted that the curves of deadtime and recovery time versus pressure are essentially straight lines. It will also be noted that the deadtime and recovery time both decrease with over-voltage. Figure 12 includes a curve of resolving time versus overvoltage for which it is assumed that the detecting circuit responds only to pulses larger than 30

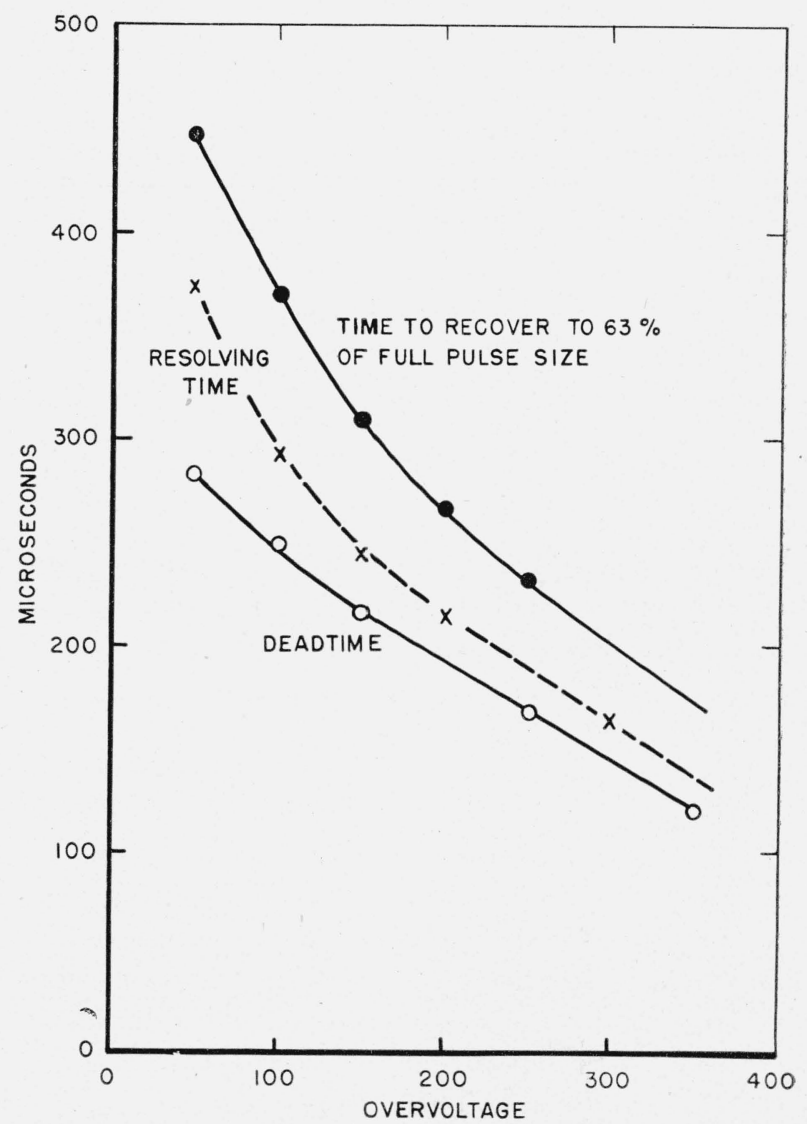

Figure 12. Variation of deadtime, resolving time, and recovery time of typical mica window argon-alcohol counter (20-cm pressure) with variation in overvoltage.

For the resolving-time curve, it is assumed that the detecting circuit responds only to pulses greater than 30 percent of the full pulse size obtained at an overvoltage of $100 \mathrm{v}$. 


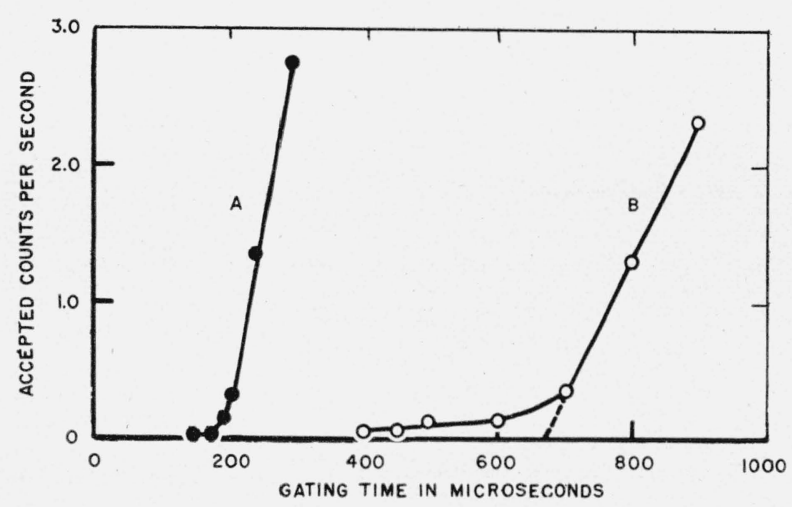

Figure 13. Accepted counting rate versus gating time of two mica-window argon-alcohol counters, identical except for gas content.

Counters filled with alcohol at a pressure of $12 \mathrm{~mm}(\mathrm{hg})$ and remainder argon. Curve A is for counter with 10-cm pressure, curve B for counter with 70-cm pressure.

percent of the normal pulse size. Since the pulse size increases with voltage, the resolving time decreases with over-voltage at a greater rate than do the deadtime and the recovery time.

Figure 13 shows curves of accepted counts per second versus gating time for two mica-window counters identical except for gas content. Curve $\mathrm{A}$ is for a counter with $10-\mathrm{cm}(\mathrm{Hg})$ total pressure, whereas curve B is for a counter with $70-\mathrm{cm}(\mathrm{Hg})$ total pressure. Since each of the counters contained alcohol at a pressure of $12-\mathrm{mm}(\mathrm{Hg})$, the high-pressure counter (curve B) had an abnormally low percentage of alcohol. It will be noted that curve B has a pronounced "tail," whereas that of curve $\mathrm{A}$ is negligible. The tail is due to the fact that, after a pulse occurs, the efficiency of a counter increases gradually from zero to its full value, as reported by Curran and Rae (see footnote 4). Because of the approximately straight-line

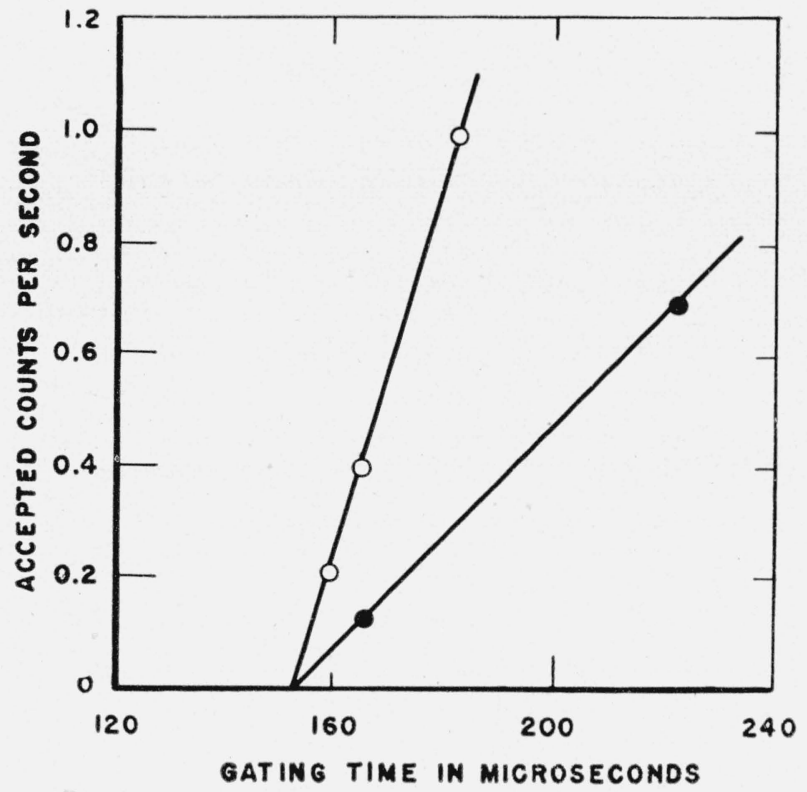

Figure 14. Accepted counts per second versus gating time for 5-percent recovery taken on a single counter at two different counting rates.

rise of efficiency, extrapolation of the straight-line portion of the curves, such as shown in figure 13, gives the same deadtime as is represented by the point at which the counter efficiency reaches half its full value.

Figure 14 shows curves of accepted counts per second versus gating time taken on a single counter at two different counting rates. The time axis intercepts give the 5-percent recovery time. Both curves give a 5-percent recovery time of 153 microseconds. This is indicative of the reproducibility obtained with the instrument.

Washington, July 9, 1948 